\title{
Accuracy and Awareness in the Perception and Categorization of Male Sexual Orientation
}

\author{
Nicholas O. Rule and Nalini Ambady \\ Reginald B. Adams Jr. \\ Tufts University \\ The Pennsylvania State University \\ C. Neil Macrae \\ University of Aberdeen
}

\begin{abstract}
For clear and unambiguous social categories, person perception occurs quite accurately from minimal cues. This article addresses the perception of an ambiguous social category (male sexual orientation) from minimal cues. Across 5 studies, the authors examined individuals' actual and self-assessed accuracy when judging male sexual orientation from faces and facial features. Although participants were able to make accurate judgments from multiple facial features (i.e., hair, the eyes, and the mouth area), their perceived accuracy was calibrated with their actual accuracy only when making judgments based on hairstyle, a controllable feature. These findings provide evidence that suggests different processes for extracting social category information during perception: explicit judgments based on obvious cues (hairstyle) and intuitive judgments based on nonobvious cues (information from the eyes and mouth area). Differences in the accuracy of judgments based on targets' controllability and perceivers' awareness of cues provides insight into the processes underlying intuitive predictions and intuitive judgments.
\end{abstract}

Keywords: judgmental accuracy, person perception, nonverbal behavior, sexual orientation, face perception

In navigating our social world, we constantly encounter new people and form impressions of them. Some aspects of these impressions may be called immediately to our attention (e.g., "that man has a gun"), but countless other aspects are perceived, encoded, and reacted to outside of our awareness (see Choi, Gray, \& Ambady, 2005; Smith, Archer, \& Costanzo, 1991). Although much attention has been paid to the issue of how we categorize and perceive others (Macrae \& Bodenhausen, 2000), most of this work has focused on the categorization and perception of individuals belonging to obvious social categories (such as race or gender), but little attention has been devoted to less obvious and more ambiguous social groups.

One such perceptually ambiguous category is that of male sexual orientation. As in the case of most ambiguous, less visible

Nicholas O. Rule and Nalini Ambady, Department of Psychology, Tufts University; Reginald B. Adams Jr., Department of Psychology, The Pennsylvania State University; C. Neil Macrae, School of Psychology, University of Aberdeen.

This research was supported in part by National Science Foundation Grant BCS-0435547 to Nalini Ambady and a National Science Foundation Graduate Research Fellowship to Nicholas O. Rule. Portions of this work were presented as part of an undergraduate honors thesis by Nicholas O. Rule to the Department of Psychological and Brain Sciences at Dartmouth College, Hanover, New Hampshire.

We thank Jason Mitchell, Elsie Wang, Malia Mason, and Oliver Hinds for their advice and assistance and Jay Conner for posing for the sample photograph.

Correspondence concerning this article should be addressed to Nicholas O. Rule, Department of Psychology, Tufts University, 490 Boston Avenue, Medford, MA 02155. E-mail: Nicholas.Rule@tufts.edu identities, individuals communicate their sexual orientation both intentionally, via cues such as clothing and hairstyle, and unintentionally, via their nonverbal behavior. Lay intuitions hold that sexual orientation can be judged from obvious cues. People report that the behaviors most telling for judgments of sexual orientation are deliberate eye gaze (Carroll \& Gilroy, 2002; Nicholas, 2004), a cue that indicates sexual interest (Mason, Tatkow, \& Macrae, 2005; see also Ekman \& Friesen, 1969), and clothing (Rudd, 1996), both of which are controllable and intentional cues. But contrary to lay intuitions, sexual orientation can also be judged at rates higher than chance from nonverbal cues that are often communicated unintentionally. For example, sexual orientation has been found to be accurately perceived from video clips (Berger, Hank, Rauzi, \& Simkins, 1987; Johnson, Gill, Reichman, \& Tassinary, 2007; Shelp, 2002) and vocal cues (Gaudio, 1994; Linville, 1998; Smyth, Jacobs, \& Rogers, 2003). Moreover, Ambady, Hallahan, and Conner (1999) showed that sexual orientation could be accurately judged from "thin slices" of nonverbal behavior, in this case from as little information as a 1-s silent video clip. In addition, they also found above-chance accuracy for still frames from the dynamic video clips, suggesting that subtle nonverbal cues may have provided information in differentiating gay individuals from straight individuals.

Here, we focused on the role of the face in communicating information about sexual orientation. Facial appearance influences judgments of gender, race, and the relative age of others (Bruce \& Young, 1998), and even individual features of the face can themselves convey social category information (e.g., gender; Brown \& Perrett, 1993). However, few studies to date have examined ambiguous identities, and none has done so with a naturally occurring, perceptually ambiguous group (see also Ostrom \& Sedikides, 
1992). Thus, the first goal of the current work was to determine whether sexual orientation can be accurately judged from static pictures of the face.

Specific facial features have been found to influence person perception to varying degrees for perceptually obvious social groups. Numerous studies have indicated that hair is a primary distinctive feature in the perception and categorization of faces (Brown \& Perrett, 1993; Ellis, Deregowski, \& Shepherd, 1975; Haig, 1986; Macrae \& Martin, 2007; Martin \& Macrae, 2007; Roberts \& Bruce, 1988; Wright \& Sladden, 2003). Studies examining the face's internal features, however, have indicated that the eyes/eyebrow region are often the most salient cues in perceiving and recognizing faces, followed by the mouth/lips region (Brown \& Perrett, 1993; Davies, Ellis, \& Shepherd, 1977; Haig, 1986; Shepherd, Davies, \& Ellis, 1981). Considering the importance, then, of hairstyle, the eye region, and the mouth area for discriminating other social categories, the second goal of the present work was to examine the role of each of these specific facial features in the accurate perception of sexual orientation.

A third goal of the present work was to examine the degree of overlap between estimations regarding the accuracy of judgments and the actual accuracy of judgments of sexual orientation from the face and facial features. Much research on social perception suggests that individuals possess very little awareness of their abilities across various domains (e.g., Ames \& Kammrath, 2004; Patterson, Foster, \& Bellmer, 2001). More often than not, people overestimate their performance, believing that they are much better at a given task than they actually are (Ames \& Kammrath, 2004). One possible reason for this inconsistency between performance and appraisal is that individuals might simply be attending to the wrong information (DePaulo, Charlton, Cooper, Lindsay, \& Muhlenbruck, 1997). For example, when judging the rapport between two targets, participants use one set of cues to make accurate judgments, but because they are not aware of what these cues are, they instead report that their judgments are based on some other set of false cues (Bernieri, Davis, Rosenthal, \& Knee, 1994). Similarly, in a meta-analysis, DePaulo et al. (1997) found that across thousands of decoders, the relationship between accuracy and confidence in detecting deception was essentially nonexistent $(r=$ .04). Thus, there appears to be little relationship between individuals' predicted and actual accuracy, especially for nonverbal behavior.

On the basis of this work, then, we expected that participants would be similarly naïve in accurately predicting their ability to judge male sexual orientation but only for some cues. Hairstyle may well be the most voluntarily controlled feature of the face. Grooming of the hair is very common, and because hair plays such a conspicuous role in the perception of individuals and groups, it also solicits perceivers' attention and awareness (e.g., Pozzulo \& Warren, 2003). Men do not typically groom their eyes and mouth as extensively as their hair. The eyes and mouth are also more likely to be outside of actors' attention and control and perceivers' awareness (see also Ekman \& Friesen, 1969). We predicted that perceivers may differ in their awareness of which cues allow for accurate judgments of sexual orientation. Specifically, we believed that perceivers would knowingly extract information about sexual orientation from targets' hairstyles, an explicitly controlled cue, but would remain unaware of their ability to accurately judge sexual orientation from the eyes and mouth.
We examined these three goals across five studies. In Study 1, participants were asked to make judgments of sexual orientation from static facial photographs. In Studies 2 and 3, we then examined the accuracy of judgments from individual features of the face to determine which cues may underlie such judgments. In Study 4, participants also made judgments from specific features but, in addition, provided postdictions of their accuracy. This allowed us to test perceivers' awareness of their ability to judge sexual orientation from specific features by measuring the calibration between their perceived accuracy and actual accuracy in making these judgments. Finally, Study 5 extended the generalizability and ecological validity of the prior results by using a second, more naturalistic, set of images.

\section{Study 1}

Does the face provide sufficient information to permit judgments of sexual orientation? In an attempt to answer this question, we asked participants to judge static images of self-identified gay and straight men.

\section{Method}

Participants. Twenty-three (11 female, 12 male) undergraduates participated for partial credit in an introductory psychology class. $^{1}$

Stimuli. The images consisted of 81 (45 gay, 36 straight) men obtained from public, online personal advertisements posted in various major U.S. cities. Targets were therefore self-defined for sexual orientation, anonymous, and available to the public domain. Images were taken from the 18-30-years-old age group on the personals websites. Only images presenting a directly oriented face free of adornments and facial hair were selected for use. The images were removed from their original context and placed onto a white background. Targets' ears and hair were retained in the cropping, but all other extra-facial information (e.g., neck) was removed (see Figure 1A). Images were then gray-scaled and standardized to 3 in. $(7.6 \mathrm{~cm}) \times 5$ in. $(12.7 \mathrm{~cm})$ in size. None of the targets' sexual orientations were disclosed to participants, no targets were obtained from the local area, and no participants reported familiarity with any of the targets.

Procedure. Participants saw all 81 images in random order using PsyScope 1.2.5 (Cohen, MacWhinney, Flatt, \& Provost, 1993). Participants were instructed that they would be seeing images of men's faces on a computer screen and were told that they were to indicate the targets' sexual orientations by clicking the mouse on one of four buttons on the screen (Very Gay, Somewhat Gay, Somewhat Straight, and Very Straight). Participants were instructed to base their opinions on what they believed "most people" or "society" would say. We did not ask for participants' direct opinions about the targets' sexual orientations so as to allay any discomfort that might arise from labeling others'

\footnotetext{
${ }^{1}$ Participants were neither screened nor questioned about their sexual orientation for any of the present studies. In addition, results from Study 1 showed no differences between male and female participants in terms of accuracy (cf Ambady et al., 1999), $\bar{r}_{\text {female }}=.31, S D=.11 ; \bar{r}_{\text {male }}=.31, S D=.15 ; t(21)=$ $.01, p=.99, r=.02$. Thus, data were collapsed across sex in all subsequent studies.
} 


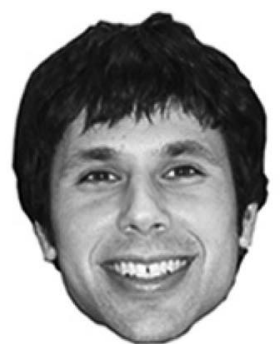

A

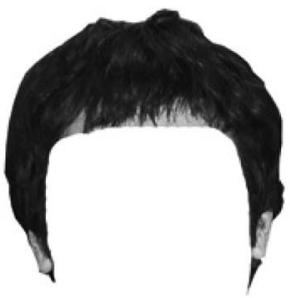

E

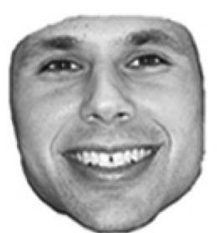

B

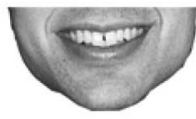

F

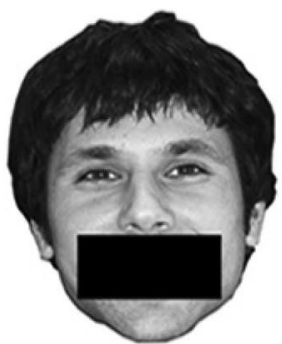

C

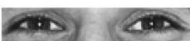

G

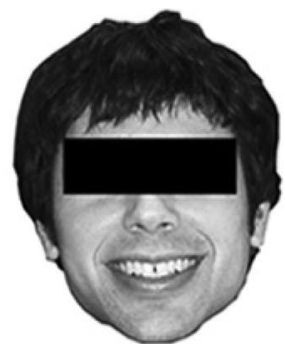

D

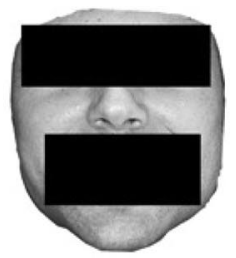

$\mathrm{H}$

Figure 1. Sample stimuli: (A) full face, Studies 1, 2, 4, and 5; (B) hair removed, Study 2; (C) mouth occluded, Study 2; (D) eyes occluded, Study 2; (E) hair only, Studies 3 and 4; (F) mouth only, Studies 3 and 4; (G) eyes only, Studies 3 and 4; and (H) all salient features removed, Study 3.

sexual orientations (a practice that is commonly regarded as socially inappropriate) and because we believed participants would answer more honestly if they did not think their responses would be regarded as their personal opinions. Participants were urged to use their "gut instinct" in rating the faces and to work as quickly as possible.

\section{Results and Discussion}

The accuracy of responses was measured using sensitivity correlations (see Judd, Ryan, \& Park, 1991). ${ }^{2}$ Thus, each participant's scores were converted to a 4-point scale from gay to straight: $1=$ very gay, $2=$ somewhat gay, $3=$ somewhat straight, and $4=$ very straight. These values were then correlated with a dichotomous vector of $0 \mathrm{~s}$ and $1 \mathrm{~s}$, coded for the targets' actual sexual orientations: $0=$ homosexual, $1=$ heterosexual. This resulted in a point-biserial correlation coefficient $(r)$ for each participant, which represented that participant's accuracy in categorizing the faces as gay or straight.

To test the significance of the sample's overall accuracy, we calculated a $95 \%$ confidence interval (CI) for the mean correlation across participants. To average the correlation coefficient, we first converted the $r$ values to Fisher's $z$ scores. ${ }^{3}$ Once these scores were averaged, we calculated the standard deviation of the $z$ transforms and the $95 \%$ CI. These values were then transformed back to correlation coefficients $(r)$ using the inverse of the Fisher's $z$ transform: ${ }^{4} \bar{r}$ $=.31, S D=.13,95 \% \mathrm{CI}=.26-.36$. Chance accuracy would be equivalent to no relationship between participants' judgments and the coded vector representing participants' actual accuracy (i.e., $r=.00$ ). As the $95 \%$ CI did not include 0 , we may conclude that participants' judgments were significantly accurate at $\alpha=.05$. Thus, participants judged male sexual orientation from the face with accuracy that was significantly better than chance.

Previous work showed that sexual orientation could be judged significantly better than chance from brief "thin slice" video clips and dynamic, full-body photographs (Ambady et al., 1999). Here, we have demonstrated that accurate judgments can be made from an even more restricted medium: still, grayscale photographs of faces. This extends the previous work and raises new questions about the cues that may underlie these judgments.

\section{Study 2}

Given that sexual orientation was judged significantly better than chance in the previous study, the goal of the present work was to examine the role of specific facial features in supporting such judgments. Participants judged the same images as in Study 1 (a) with no alterations, (b) with the hair removed, (c) with the mouth occluded, and (d) with the eyes and brows occluded.

\section{Method}

Participants. Twenty-nine paid undergraduate participants (15 female, 14 male) saw all 324 images ( 81 photos $\times 4$ conditions) in a randomized, repeated-measures design.

${ }^{2}$ The mean response latency for judgments of sexual orientation across all of the studies reported in this paper was $2.61 \mathrm{~s}(S D=1.22)$.

$$
\begin{aligned}
& { }^{3} \text { Fisher's } z=\frac{1}{2} \times \log _{e}\left[\frac{(1+r)}{(1-r)}\right] . \\
& { }^{4} r=\frac{e^{2 z}-1}{e^{2 z}+1} .
\end{aligned}
$$


Stimuli and procedure. Each of the 81 images used in Study 1 was modified using Adobe Photoshop 5.5 to remove the hair, cover the mouth and lips, and cover the eyes and brows. This resulted in four conditions (control, hair-removed, mouthoccluded, and eyes-occluded), producing a total of 324 images (see Figures 1A, 1B, 1C, and 1D, respectively). Images were presented randomly and rated using the same instructions and four-choice paradigm as in Study 1.

\section{Results and Discussion}

Stimuli per condition. Although participants viewed each of the faces across all four conditions, we used only data from the first trial in which a given participant saw each of the faces in the analysis to prevent carryover effects within a stimulus across conditions. As the conditions were drawn randomly for each stimulus, participants saw approximately equal numbers of faces in each of the four conditions: $M_{\text {full face }}=20.52, S D=5.27$; $M_{\text {hair-removed }}=20.71, S D=4.71 ; M_{\text {mouth-occluded }}=20.39, S D=$ 4.21; and $M_{\text {eyes-occluded }}=19.36, S D=4.36$. In addition, a 2 $($ Sexual Orientation) $\times 4$ (Feature) repeated-measures analysis of variance (ANOVA) showed neither a main effect of feature, $F(3$, $84)=.54, p=.66, \eta^{2}=.01$, nor a Feature $\times$ Sexual Orientation interaction for the frequency of trials in each of the conditions, $F(3,84)=1.67, p=.18, \eta^{2}=.35$.

Accuracy. As in Study 1, participants' ratings of each face were converted to a 4-point numerical scale and measured using sensitivity correlations. Measures of accuracy were obtained for each participant in each of the four conditions (full face, hairremoved, mouth-occluded, and eyes-occluded). We then calculated the mean, standard deviation, and confidence interval for participants' sensitivity correlations in each condition: $\bar{r}_{\text {full face }}=.32, S D=$ $.27,95 \% \mathrm{CI}=.22-.42 ; \bar{r}_{\text {hair-removed }}=.19, S D=.20,95 \% \mathrm{CI}=$ $.12-.26 ; \bar{r}_{\text {mouth-occluded }}=.22, S D=.26,95 \% \mathrm{CI}=.13-.31$; and $\bar{r}_{\text {eyes-occluded }}=.26, S D=.33,95 \% \mathrm{CI}=.14-.37$. As the mean sensitivity correlations for each of the four conditions were within 95\% CIs that do not include 0 , we again concluded that participants' accuracy was significantly better than chance. To investigate whether accuracy varied by condition, we conducted a one-way repeated-measures ANOVA on the participants' Fisher's $z$-transformed sensitivity correlations for the four conditions. The results of the ANOVA showed no differences in accuracy, $F(3$, 28) $=1.05, p=.39, \eta^{2}=.10$.

Removing a single feature from a man's face therefore did not significantly impair perceivers' ability to accurately judge his sexual orientation. Thus, these data indicate that no single facial feature is necessary for the judgment of male sexual orientation. Rather, information may be communicated via multiple features of the face. Study 3 explored this further by examining judgments made for each of these features in isolation.

\section{Study 3}

Study 2 showed that the removal of a single facial feature does not significantly impair the accuracy of judgments of male sexual orientation. Hence, we may conclude that none of these features is itself vital to the judgment of sexual orientation from the male face. But this does not mean that each of these salient features is not important in making accurate judgments. Thus, in Study 3, we attempted to answer the question as to which feature or features might be diagnostic in judgments of male sexual orientation by isolating each of the salient features. Participants were therefore asked to make judgments of sexual orientation on the basis of information from the hair only, eyes only, or mouth area only. Finally, a separate group of participants judged sexual orientation when all of these features were removed.

\section{Method}

Participants. A total of 98 undergraduates (56 female, 42 male) made judgments of each of the targets' faces in exchange for partial course credit in an introductory psychology class. Participants completed one of four conditions: rating just targets' hairstyles $(n=25)$, rating just targets' mouth areas $(n=26)$, rating just targets' eyes $(n=24)$, or rating targets' faces without any of these salient features (described below; $n=23$ ).

Stimuli. As Studies 1 and 2 had an unequal number of gay and straight targets, we conducted a web search of online personal advertisements, using the same procedures as in Study 1, to acquire 9 additional heterosexual male targets, for a total of 45 gay targets and 45 straight targets. We then modified each of the targets using Adobe Photoshop CS to produce each of the four conditions: hair only, mouth only, eyes only, and with no salient features.

In the hair-only condition, faces were erased from the images and cropped so as to show the hair, ears, sideburns (if present) and some skin from the forehead (see Figure 1E). In the mouth-areaonly condition, the lower halves of the faces were cropped just below the tip of the nose (see Figure 1F). In the eyes-only condition, the eyes were cropped below the eyebrows and as wide as the outer canthi of the eyes (see Figure 1G). Finally, the fourth condition removed each of these salient features from the faces. Similar to the stimulus-preparation procedures in Study 2, we simultaneously removed the hair, covered the mouth, and covered the eyes of each of the target faces. This left visible only the target's nose, cheeks, face shape, and skin texture (see Figure $1 \mathrm{H}$ ). This last condition, therefore, served as a control condition against which to test the relevance of the three previously hypothesized salient cues.

To address the possibility that differences in affect between the gay and straight targets might influence participants' judgments of sexual orientation, we had a group of three experimentally blind research assistants code each of the unaltered faces, on the basis of affective expression, into three categories: neutral, happy, or other. Frequencies for their categorizations are presented in Table 1. A chi-square test showed that the gay and straight targets did not significantly differ in expression, $\chi^{2}(2, N=90)=.24, p=.89$, $V_{\text {Cramer }}=.05$. Intensity of affect was also rated by two independent samples of naïve participants. One sample rated each of the faces

Table 1

Distribution of Facial Expressions by Count for Gay and Straight Targets in Study 3

\begin{tabular}{lccc}
\hline Target sexual orientation & Neutral & Happy & Other \\
\hline Gay $(n=45)$ & 11 & 28 & 6 \\
Straight $(n=45)$ & 16 & 20 & 9 \\
\hline
\end{tabular}


categorized as "happy" on a scale from 1 (a little happy) to 7 (very happy) for the mouth-only condition $(n=15)$. The other sample made the same ratings for the eyes-only condition $(n=15)$. Scores were averaged across participants for each target and compared via independent-samples $t$ tests. No differences were found in the intensity of happiness expressed by the gay and straight targets for either the mouth-area-only condition, $t(46)=1.73, p=.09, r=$ .25 , or eyes-only condition, $t(46)=.38, p=.70, r=.06$, indicating that the gay and straight targets did not significantly differ in affective expression.

In addition, 25 undergraduates rated the targets for attractiveness along a 7-point scale, as part of a larger set of faces being rated. Attractiveness ratings for each face were averaged across participants and compared via an independent-samples $t$ test, which showed no differences between the gay and straight targets, $t(88)=1.37, p=.18, r=.14$.

Procedure. Participants saw all 90 images for their assigned condition in random order using DirectRT (Jarvis, 2004). Participants were instructed that they would be seeing images of men's faces on a computer screen and were told that they were to rate the targets' probable sexual orientations along a 7-point scale $(1=$ very gay, $7=$ very straight . As in Studies 1 and 2, participants were instructed to base their opinions on what they believed "most people" or "society" would say and to use their "gut instinct" in making their judgments.

\section{Results and Discussion}

Sensitivity correlations. Sensitivity correlations revealed that accuracy was significantly better than chance in the haironly $(\bar{r}=.24, S D=.13,95 \% \mathrm{CI}=.19-.29)$, mouth-area-only $(\bar{r}=.15, S D=.10,95 \% \mathrm{CI}=.11-.18)$, and eyes-only $(\bar{r}=$ $.12, S D=.11,95 \% \mathrm{CI}=.08-.16)$ conditions. Accuracy was not significantly better than chance in the condition without these salient features, however, $\bar{r}=.00, S D=.13,95 \% \mathrm{CI}=-.05-.06$.

Similar to Study 2, we then compared Fisher's z-transformed accuracy scores for the four conditions via between-participants univariate ANOVA. The omnibus test showed that differences existed between the four conditions, $F(3,94)=16.89, p<.001, \eta^{2}=.35$. Pairwise, Bonferroni-corrected $(\alpha=.0083) t$ tests showed that this effect was driven by significantly greater accuracy for judgments based on the salient features versus judgments when the salient features were simultaneously removed (see Table 2). In addition, judgments based on hair only were significantly more accurate than those based on the eyes only or mouth area only.

Table 2

Bonferroni-Corrected $(\alpha=.0083)$, Independent-Samples $t$ Values (Degrees of Freedom in Parentheses) for Two-Tailed Comparisons of the Accuracy Judgments in Study 3

\begin{tabular}{|c|c|c|c|c|}
\hline Condition & Hair only & Mouth area only & Eyes only & $\begin{array}{l}\text { No salient } \\
\text { features }\end{array}$ \\
\hline Hair only & & $2.97^{*}(49)$ & $3.56^{*}(47)$ & $6.27^{*}(46)$ \\
\hline Mouth area only & & & 0.87 (48) & $4.21^{*}(47)$ \\
\hline Eyes only & & & & $3.35^{*}(45)$ \\
\hline
\end{tabular}

Thus, similar to previous work on the perception of perceptually obvious groups (e.g., Brown \& Perrett, 1993; MacLin \& Malpass, 2001), both controllable and relatively uncontrollable features of the face contributed to accurate judgments of male sexual orientation. Hairstyle, for example, is a deliberate aspect of appearance that is groomed to look a particular way. It is no surprise that style-based components of appearance might reveal information about cultural subgroups. Indeed, Rudd (1996) showed that clothing style contributes to perceptions of male sexual orientation. But even more interesting are the findings that judgments of sexual orientation can be made from information as limited as the eyes, an area not commonly subject to grooming or controllable displays of identity. This raises the important question of whether perceivers use some of these cues differently than they do others when making their judgments of sexual orientation. Study 4 explored this possibility in more detail.

\section{Study 4}

During debriefing in the previous studies, the majority of participants indicated that they had been guessing throughout the experiment. Many felt exasperated, expressing that they had "no idea" of how to make these judgments and believed that their performance would be quite low. Participants' low confidence in their ability to judge sexual orientation, in light of their abovechance accuracy, is intriguing and represents a deviation from much research in social cognition, in which participants tend to express overconfidence in their performance (e.g., DePaulo et al., 1997). Thus, the goal of Study 4 was to explicitly test the relationship between individuals' perceived accuracy and their actual accuracy in making judgments of sexual orientation based on the full face and individual features.

\section{Method}

Participants. Forty-nine undergraduates (29 female, 20 male) participated for partial course credit in an introductory psychology class.

Stimuli. The stimuli consisted of the same 90 faces used in Study 3 . Thirty images (15 gay men, 15 straight men) from each of the salient features conditions (hair only, mouth area only, and eyes only) were presented to participants in a counterbalanced fashion. Specifically, participants rated 30 images from the hair-only condition, a different 30 images from the moutharea-only condition, and the remaining 30 images from the eyes-only condition. The images were blocked by feature and presented in random order within the blocks, which were also randomly ordered. Finally, participants rated all 90 of the full (unaltered) faces. Therefore, each target was rated twice: first based on only one feature and second based on the full face. Hence, the experiment consisted of four repeated-measures conditions, with counterbalanced presentation of targets in the first three conditions.

Procedure. Instructions for judgments of the faces were the same as in Study 3, and participants made ratings on the same 7-point scale. At the end of each section, however, participants were prompted by a computer screen that asked them to indicate how accurate they believed their judgments were in the previous section: $0-100 \%$. Because 30 unique targets were used for each of 
the feature-based sections, we created three versions of the experiment to counterbalance the representation of targets across the three features; the number of participants per version was approximately equal.

\section{Results}

Actual accuracy. Mean accuracy, standard deviations, and 95\% CIs for each of the four conditions were calculated using sensitivity correlations, as above. Accuracy was again significantly better than chance for each of the salient features and for the full faces, $\bar{r}_{\text {hair only }}=$ $.27, S D=.21,95 \% \mathrm{CI}=.22-.34 ; \bar{r}_{\text {mouth area only }}=.11, S D=.17$, $95 \% \mathrm{CI}=.06-.16 ; \bar{r}_{\text {eyes only }}=.11, S D=.19,95 \% \mathrm{CI}=.06-.17$; $\bar{r}_{\text {full face }}=.31, S D=.11,95 \% \mathrm{CI}=.27-.34$.

Fisher's z-transformed accuracy scores were compared in a one-way, repeated-measures ANOVA. The omnibus test showed a significant difference across the four conditions, $F(3,144)=$ 22.73, $p<.001, \eta^{2}=.23$. Bonferroni-corrected $(\alpha=.0083)$, paired-samples $t$ tests indicated that this effect was supported by significantly greater accuracy for judgments based on the full face and hair only conditions, compared with judgments based on the eyes only and mouth area only (see Table 3). However, the hair-only and full-face conditions did not differ in accuracy.

Postdicted accuracy. Participants' postdicted accuracy was averaged for each of the four conditions, $M_{\text {hair only }}=40.69 \%, S D=$ $21.54 \% ; M_{\text {mouth area only }}=38.64 \%, S D=24.24 \% ; M_{\text {eyes only }}=$ $35.84 \%, S D=22.10 \% ; M_{\text {full face }}=45.03 \%, S D=21.98 \%$. A one-way, repeated-measures ANOVA showed that participants' postdicted accuracy differed by condition, $F(3,144)=4.87, p<.01$, $\eta^{2}=.02$. Bonferroni-corrected $(\alpha=.0083)$, paired-samples $t$ tests showed that this effect was due to significant differences in participants' postdicted accuracy between the full-face condition versus the eyes-only condition, $t(48)=3.73, p<.001, r=.47$. No other comparisons differed significantly (all $t \mathrm{~s}<2.67, p \mathrm{~s}>.01, r \mathrm{~s}<.36$ ).

Actual accuracy versus postdicted accuracy. To measure the relationship between actual and perceived accuracy, we correlated the Fisher's $z$ transforms of the participants' actual accuracy with that of their postdicted accuracy for each of the four conditions. This revealed that participants were inaccurate in postdicting their accuracy in the full-face condition, $r(47)=.07, p=.50$; eyes-only condition, $r(47)=-.04, p=.78$; and mouth-area-only condition, $r(47)=-.02, p=.89$. However, participants were accurate in postdicting their accuracy in the hair-only condition, $r(47)=.31$, $p<.05$.

We compared these effects following the procedures for testing differences between correlated, nonoverlapping correlations outlined by Olkin and Finn (1990). The omnibus test showed no

Table 3

Bonferroni-Corrected ( $\alpha=.0083)$, Paired-Samples $t$ Values (All $d f s=48)$ for Two-Tailed Comparisons of the Accuracy

Judgments in Study 4

\begin{tabular}{lcccc}
\hline \multicolumn{1}{c}{ Condition } & Hair only & Mouth area only & Eyes only & Full face \\
\hline Hair only & $5.11^{*}$ & $5.03^{*}$ & 1.17 \\
Mouth area only & & 0.09 & $7.56^{*}$ \\
Eyes only & & & $7.01^{*}$ \\
\hline
\end{tabular}

${ }^{*} p<.0083$. overall differences between the correlations for each condition, $\chi^{2}(3, N=49)=3.32, p=.34$. But planned contrasts showed that the relationship between actual and postdicted accuracy for judgments based on hairstyle (a) was significantly greater than the relationship between actual and postdicted accuracy for judgments based on eyes only, $\chi^{2}(1, N=49)=4.08, p=.04$, (b) was marginally greater than the relationship between actual and postdicted accuracy for judgments based on the mouth area only, $\chi^{2}(1$, $N=49)=3.69, p=.05$, and (c) did not significantly differ from the relationship between actual and postdicted accuracy for judgments based on the full face, $\chi^{2}(1, N=49)=1.71, p=.19$. Notably, the relationship between actual and postdicted accuracy for judgments based on the full face did not significantly differ from the relationship between actual and postdicted accuracy for judgments based on the eyes only, $\chi^{2}(1, N=49)=0.34, p=.56$, or mouth area only, $\chi^{2}(1, N=49)=0.22, p=.64$.

Therefore, the relationship between actual and postdicted accuracy for judgments based on full faces is substantially weaker than it is for judgments based on hairstyle alone, stronger than it is for judgments based on the eyes and mouth area, but not significantly different from any of these. It is possible that this is the result of the combination of hairstyle (an accurately judged, obvious cue) with the eyes and mouth area (accurately judged, nonobvious cues). We discuss this in further detail below.

Manipulation check. Comparisons of participants' ratings of the features versus full faces may be problematic because the stimuli are repeated. Although in many cases it may be difficult to match particular, isolated features with the full (parent) faces during a second presentation, the full-face judgments may nevertheless have been biased because of repetition.

Therefore, we had a separate sample of 22 undergraduates (17 female, 5 male) rate the full faces in exchange for partial course credit in an introductory psychology class. Targets were judged on the same 7-point scale as in the main study and, after completing the ratings, participants were prompted by the computer to postdict their accuracy.

The data were analyzed using sensitivity correlations and showed accuracy that was significantly better than chance $\left(M_{\text {Actual accuracy }}=.33, S D=.12,95 \% \mathrm{CI}=.20-.44\right)$. An independent-samples $t$ test comparing these accuracy scores with those for the full-face condition from the main experiment showed no significant difference, $t(69)=.53, p=.60, r=.06$. In addition, a series of Bonferroni-corrected $(\alpha=.017)$, independent-samples $t$ tests replicated the differences in accuracy between the full face and individual features, as observed in the main experiment: hair only, no difference, $t(68)=1.33$, $p=.19, r=.16$; mouth area only, significantly different, $t(68)=6.05, p<.001, r=.59$; and eyes only, significantly different, $t(68)=5.70, p<.001, r=.57$.

Parallel effects were also found for postdicted accuracy. Postdicted accuracy did not differ from the full-face condition in the main experiment $\left(M_{\text {Postdicted accuracy }}=48.00 \%, S D=16.99 \%\right)$, $t(69)=.55, p=.58, r=.07$, and Bonferroni-corrected $(\alpha=.017)$ $t$ tests showed a significant difference against the eyes-only condition, $t(68)=2.44, p=.017, r=.28$. All other comparisons were nonsignificant (all $t \mathrm{~s}<1.80, p \mathrm{~s}>.08, r \mathrm{~s}<.21$ ).

Finally, meta-analytic comparisons showed that the relationships between actual and postdicted accuracy did not significantly differ between the independent sample here, $r(20)=.17, p=.44$, 
and the dependent sample from the main experiment $(Z=.38, p=$ $.35){ }^{5}$ Thus, we can reasonably conclude that repetition of the targets did not significantly bias participants' judgments in the main experiment, because a second, independent sample showed statistically congruent effects.

\section{Discussion}

Study 4 replicated the findings of Study 3 by demonstrating that the three salient features independently allow for above-chance accuracy in judging male sexual orientation. As in Study 3, accuracy based on judgments from the hairstyle was significantly greater than accuracy based on judgments from the eyes only and mouth area only. In addition, Study 4 provided new data with which to compare the accuracy of judgments based on features with the accuracy of judgments based on the full face. These data showed that, like hairstyle, accuracy from judgments based on the full face was significantly greater than accuracy based on judgments from the eyes only and mouth area only. The accuracy of judgments based on the full face did not significantly differ from the accuracy of judgments based on hairstyle.

Participants in Study 4 also provided postdicted estimates of their accuracy for judgments based on the salient features and full face. There was some variance among these postdicted assessments: Participants perceived their accuracy to be significantly greater based on the full face, compared with the eyes only.

More interesting, however, was the relationship between participants' postdicted accuracy and their actual accuracy. When judging male sexual orientation based on hairstyle, participants' postdicted accuracy was significantly calibrated with their actual accuracy. When judging male sexual orientation based on the eyes or mouth area, participants' postdicted accuracy showed virtually no relationship with their actual accuracy. Finally, when judging male sexual orientation from full faces, which incorporate all three of these salient features, participants' postdicted accuracy was not significantly calibrated with their actual accuracy but was greater than the relationships between actual and postdicted accuracy for judgments based on the eyes only and mouth area only.

Participants were therefore able to postdict their accuracy when making judgments based on hairstyle but not for any of the other conditions (i.e., eyes only, mouth area only, and full face). One explanation for this may be that participants hold particular stereotypes about gay and straight men's hairstyles that are somewhat accurate (although imperfect). Participants' postdicted accuracy could therefore track with their actual accuracy as a function of applying these stereotypes. If participants had stereotypes about hairstyles but not about eyes or mouths, this would explain the differences between the features.

Indeed, previous research has indicated that perceivers are differentially aware of their use of various nonverbal cues. Ekman and Friesen (1969) distinguished between cues that communicate nonverbal information explicitly and those that leak through unintentionally and are picked up by perceivers without their awareness. Hairstyle may be an obvious cue: It is intentionally styled, static, and highly descriptive. In contrast, information about sexual orientation from the eyes and mouth area may be subtle, nonobvious, and support only intuitive judgments. Perceivers could then accurately judge sexual orientation from these cues but remain unaware of their ability to do so. This agrees with previous work that has shown that individuals have low confidence in their intuitive judgments (Ambady, Bernieri, \& Richeson, 2000; Choi et al., 2005; Patterson et al., 2001).

But if perceivers knowingly judge male sexual orientation from hairstyles, why do they not postdict their judgments from full faces, which also contain hairstyles? This, too, might be explained by differences between obvious (hairstyle) and nonobvious (eyes and mouth area) cues. Specifically, participants' intuitive judgments based on the eyes and mouth area may conflict with their explicit stereotypes about particular hairstyles. Competition between the features may therefore compromise perceivers' awareness of their accuracy, because the confidence based on the obvious cue (hairstyle) may be undermined by the lack of confidence that stems from the nonobvious cues (eyes and mouth area). Indeed, the strength of relationship between participants' actual and postdicted accuracy was significant for judgments based on only the obvious cue, declined when nonobvious cues were introduced via the full face, and declined further when participants judged only the nonobvious cues.

Therefore, although participants' categorizations based on the full face are still accurate, and their estimated accuracy is not as low as for the nonobvious features, it does not correspond to their actual ability. Thus, sexual orientation appears to be perceived largely without individuals' awareness. Only in the case of highly explicit cues (in this case, hairstyle) are perceivers aware of their ability to accurately decode others' sexual orientation.

\section{Study 5}

Thus far, all of the above studies have used the same set of targets, whose images were downloaded from online personal advertisements. It is possible that differences in self-presentation may have led to some systematic differences in the appearance of gay and straight men when posting photos of themselves on personal advertisements. Therefore, in Study 5, we tested the validity of the previous results by using a sample of photos that was more controlled for demographic variables and less subject to self-presentation demands as a means to investigate the generalizability of the previous findings.

\section{Method}

Participants. Twenty-nine undergraduates (17 female, 12 male) participated for partial course credit in an introductory psychology class. Participants were naïve to the origin and identities of the targets and none reported any familiarity with any of the persons in the photographs.

Stimuli and procedure. Images were downloaded from http:// www.facebook.com. Images were from the same private university in the northeast United States. Thus, targets were relatively homogeneous in age, socioeconomic status, education, and style of dress/ appearance, and the groups were matched for racial composition. The

\footnotetext{
${ }^{5}$ Rosenthal and Rosnow (2007, p. 672) provided a formula for comparing $r$ statistics: $Z=\frac{z_{1}-z_{2}}{\sqrt{\frac{1}{N_{1}-3}+\frac{1}{N_{2}-3}}}$, where $z_{1}$ and $z_{2}$ represent Fisher's $z$ transforms of $r_{1}$ and $r_{2}, N_{1}$ and $N_{2}$ correspond to the size of the respective samples, and $Z$ refers to the standard normal.
} 
only ostensible difference between the gay and straight targets was their sexual orientation. This worked to control for any variance between the gay and straight targets that might be the result of demographic differences.

We also controlled for self-presentation by using images of gay and straight individuals that were posted on others' profiles, rather than on their own profiles. Facebook allows users to store electronic photo albums. When an individual's name is in the caption of a photo, a link is created to that person's profile without his or her consent and then appears on his or her profile. We followed these links to others' photo albums to select images that targets did not choose to post themselves. Moreover, to control for the possibility that targets' friends might post images of them that exaggerate salient aspects of their identity (e.g., caricatures of their sexual orientation), we selected only photos that contained multiple individuals. Thus, any bias in image selection would be diffused across the individuals in the photograph. Further, these album photos are often candid, "real life" photos (i.e., absent the target's awareness that the photo is being taken), rather than posed shots, thereby increasing the ecological validity of the photographs.

To obtain these photos, we performed a search for men's profiles that indicated romantic or sexual interest in other men (i.e., self-identified homosexual men). Of these profiles, we randomly selected a subset of those who had photos in other users' photo albums that contained multiple individuals in the photograph. We then selected photos using the same search criteria used in Study 1 and followed the same procedures for standardizing the faces as in Study 1. This process was then repeated for the profiles of men indicating romantic or sexual interest in women (i.e., selfidentified heterosexual men). Persons indicating interest in members of both sexes (i.e., self-identified bisexual men) were not included. This resulted in a total of 134 targets (69 gay and 64 straight), a number 1.5 times the size of the previous set used in Studies 1-4.

Participants were instructed to make judgments along the same 7-point scale used in Studies 3 and 4 and to base their judgments on how they believed most people in society would judge a given target. Participants and targets were not from the same university. Consequently, no participants reported familiarity with any of the targets.

\section{Results and Discussion}

Data were again analyzed using sensitivity correlations. As before, mean accuracy was significantly greater than chance $(\bar{r}=.22, S D=.08$, $95 \% \mathrm{CI}=.19-.24)$. Meta-analytic comparisons of full-face judgments from Study $1(Z=.33, p=.37)$ and Study $4\left(Z_{\text {dependent sample }}=.39\right.$, $p=.35 ; Z_{\text {independent sample }}=.39, p=.35$ ) suggest that the effects for targets from personal advertisements and from Facebook did not significantly differ. Therefore, the present results support the validity of the above effects and show that sexual orientation may be accurately perceived from male targets' faces in the absence of the self-presentation demands of personal advertisements.

\section{General Discussion}

Previous research on the perception and categorization of individuals has almost exclusively focused on perceptually obvious groups. Here, however, we showed that we can accurately categorize group members when the distinction between them is perceptually ambiguous. Across these studies, male sexual orientation was extracted with accuracy that was significantly better than chance from static photographs of faces. In addition, individual features of the face independently communicated information that allowed for accurate judgments. Cues from hairstyle, the mouth area, and the eyes (without brows and cropped to the outer canthi, so that not even "crows' feet" wrinkles were visible) allowed perceivers to accurately judge male sexual orientation.

But although information about male sexual orientation may have been expressed via multiple facial features, judgments from hairstyles were significantly more accurate than judgments from the eyes or mouth area. Perhaps more interesting, perceivers accurately estimated their accuracy when making judgments from the hair but not from either the eyes or mouth. This difference in knowledge about the use of some cues over others suggests that lay intuitions about the accuracy of predictions vary according to the cues being assessed.

In light of previous research (e.g., Ekman \& Friesen, 1969), it is reasonable to assume that some cues would be processed explicitly and other cues processed intuitively. Hairstyle, which is deliberately styled, might represent an explicit cue to male sexual orientation. In contrast, the eyes and mouth area are not explicitly styled or controlled in the way that hairstyle is. Information about sexual orientation from the eyes and mouth area may therefore be more subtle and processed by perceivers at an intuitive level, without awareness. This therefore suggests two paths to perceiving and categorizing social groups: (a) judgments based on explicit/ obvious cues and (b) judgments based on intuitive/nonobvious cues.

Indeed, judgments of the full faces (where both types of cues necessarily coexist) show evidence of both processes. The accuracy of judgments from full faces is equivalent to those from hairstyle, the most obvious cue. But the subjective experience of judging full faces (i.e., participants' postdicted estimations of their accuracy) was similar to that observed from judgments of the eyes and mouth area, the less obvious cues. This indicates that when participants judged the full face, they were probably not simply attending to the obvious cue to make their judgments. Rather, they might have experienced some interference from the nonobvious cues.

This observation of differential utility across facial features may provide a helpful perspective to our understanding of feature-based accounts of social categorization. Previous research has shown that much information about identity, gender, and emotion can be gleaned from facial features (e.g., Schyns, Bonnar, \& Gosselin, 2002; Vinette, Gosselin, \& Schyns, 2004). Individual features can convey decisive information about distinct emotions (e.g., Smith, Cottrell, Gosselin, \& Schyns, 2005), and category-revealing information from particular features is known to prime information about social groups, influencing subsequent memory (Mason, Cloutier, \& Macrae, 2006; Macrae \& Martin, 2007; Martin \& Macrae, 2007). Here, we have shown that group-diagnostic identifications may occur from multiple cues within a face but that some of these cues are more functional than others and may be employed distinctly by perceivers. Thus, the current data add to the theoretical understanding of social categorization by showing that 
different construal processes may lead to the same categorization outcomes through potentially alternate cognitive-perceptual paths.

By examining a perceptually ambiguous group, such as male sexual orientation, we may be in a unique position to observe these two processes as they occur within the same, naturally occurring faces at ostensibly the same time. For instance, judgments of race are based on both skin tone and other features (e.g., Blair, Judd, Sadler, \& Jenkins, 2002; Maddox, 2004). We may have theories about some cues that we can easily apply (such as skin tone), but we might not realize that other cues affect our judgments as well (such as facial features; see Blair, Judd, \& Fallman, 2004). In the case of male sexual orientation, the cues are more subtle than that of skin tone, and an examination of the use of these cues provides a nice means of dissociating the explicit and the intuitive processes that occur in person perception. Future studies may wish to examine what aspects of these features lead to accurate judgments, what their origins might be, and how we acquire the ability to detect them.

In summary, these data provide new insights as to accurate and inaccurate intuitions in person peception. The data suggest that we perceive others using two types of cues: obvious/explicit cues and nonobvious/intuitive cues. We are aware of and are well calibrated in our use of explicit cues to guide our judgments. We are not aware of and are poorly calibrated in our use of nonobvious, subtle cues that guide our judgments.

\section{References}

Ambady, N., Bernieri, F. J., \& Richeson, J. A. (2000). Toward a histology of social behavior: Judgmental accuracy from thin slices of the behavioral stream. In M. P. Zanna (Ed.), Advances in experimental social psychology (Vol. 32, pp. 201-271). San Diego, CA: Academic Press.

Ambady, N., Hallahan, M., \& Conner, B. (1999). Accuracy of judgments of sexual orientation from thin slices of behavior. Journal of Personality and Social Psychology, 77, 538-547.

Ames, D. R., \& Kammrath, L. K. (2004). Mind-reading and metacognition: Narcissism, not actual competence, predicts self-estimated ability. Journal of Nonverbal Behavior, 28, 187-209.

Berger, G., Hank, L., Rauzi, T., \& Simkins, L. (1987). Detection of sexual orientation by heterosexuals and homosexuals. Journal of Homosexuality, 13, 83-100.

Bernieri, F. J., Davis, J. M., Rosenthal, R., \& Knee, C. R. (1994). Interactional synchrony and rapport: Measuring synchrony in displays devoid of sound and facial affect. Personality and Social Psychology Bulletin, 20, 303-311.

Blair, I. V., Judd, C. M., \& Fallman, J. L. (2004). The automaticity of race and Afrocentric facial features in social judgments. Journal of Personality and Social Psychology, 87, 763-778.

Blair, I. V., Judd, C. M., Sadler, M. S., \& Jenkins, C. (2002). The role of Afrocentric features in person perception: Judging by features and categories. Journal of Personality and Social Psychology, 83, 5-25.

Brown, E., \& Perrett, D. I. (1993). What gives a face its gender? Perception, 22, 829-840.

Bruce, V., \& Young, A. (1998). In the eye of the beholder: The science of face perception. London: Oxford University Press.

Carroll, L., \& Gilroy, P. J. (2002). Role of appearance and nonverbal behaviors in the perception of sexual orientation among lesbians and gay men. Psychological Reports, 91, 115-122.

Choi, Y. S., Gray, H. M., \& Ambady, N. (2005). The glimpsed world: Unintended communication and unintended perception. In R. R. Hassin, J. S. Uleman, \& J. A. Bargh (Eds.), The new unconscious (pp. 309-333). New York: Oxford University Press.
Cohen, J. D., MacWhinney, B., Flatt, M., \& Provost, J. (1993). PsyScope: A new graphic interactive environment for designing psychology experiments. Behavioral Research Methods, Instruments, and Computers, 25, 257-271.

Davies, G., Ellis, H., \& Shepherd, J. (1977). Cue saliency in faces as assessed by the "Photofit" technique. Perception, 6, 263-269.

DePaulo, B. M., Charlton, K., Cooper, H., Lindsay, J. J., \& Muhlenbruck, L. (1997). The accuracy-confidence correlation in the detection of deception. Personality and Social Psychology Review, 1, 346-357.

Ekman, P., \& Friesen, W. V. (1969). Nonverbal leakage and clues to deception. Psychiatry, 32, 88-106.

Ellis, H. D., Deregowski, J. B., \& Shepherd, J. W. (1975). Descriptions of White and Black faces by White and Black subjects. International Journal of Psychology, 10, 119-123.

Gaudio, R. P. (1994). Sounding gay: Pitch properties in the speech of gay and straight men. American Speech, 69, 30-57.

Haig, N. D. (1986). Exploring recognition with interchanged facial features. Perception, 15, 235-247.

Jarvis, B. G. (2004). DirectRT (Version 2004.3.27) [Computer software]. New York: Empirisoft.

Johnson, K. L., Gill, S., Reichman, V., \& Tassinary, L. G. (2007). Swagger, sway, and sexuality: Judging sexual orientation from body motion and morphology. Journal of Personality and Social Psychology, 93, 321-334.

Judd, C. M., Ryan, C. S., \& Park, B. (1991). Accuracy in the judgment of in-group and out-group variability. Journal of Personality and Social Psychology, 61, 366-379.

Linville, S. E. (1998). Acoustic correlates of perceived versus actual sexual orientation in men's speech. Folia Phoniatrica et Logopaedica, 50, $35-48$.

MacLin, O. H., \& Malpass, R. S. (2001). Racial categorization of faces: The ambiguous race face effect. Psychology, Public Policy, and Law, 7, 98-118.

Macrae, C. N., \& Bodenhausen, G. V. (2000). Social cognition: Thinking categorically about others. Annual Review of Psychology, 51, 93-120.

Macrae, C. N., \& Martin, D. (2007). A boy primed Sue: Feature-based processing and person construal. European Journal of Social Psychology, 37, 793-805.

Maddox, K. B. (2004). Perspectives on racial phenotypicality bias. Personality and Social Psychology Review, 8, 383-401.

Martin, D., \& Macrae, C. N. (2007). A face with a cue: Exploring the inevitability of person categorization. European Journal of Social Psychology, 37, 806-816.

Mason, M. F., Cloutier, J., \& Macrae, C. N. (2006). On construing others: Category and stereotype activation from facial cues. Social Cognition, $24,540-562$.

Mason, M. F., Tatkow, E. P., \& Macrae, C. N. (2005). The look of love: Gaze shifts and person perception. Psychological Science, 16, 236-239.

Nicholas, C. L. (2004). Gaydar: Eye-gaze as identity recognition among gay men and lesbians. Sexuality \& Culture, 8, 60-86.

Olkin, I., \& Finn, J. D. (1990). Testing correlated correlations. Psychological Bulletin, 108, 330-333.

Ostrom, T. M., \& Sedikides, C. (1992). Out-group homogeneity effects in natural and minimal groups. Psychological Bulletin, 112, 536-552.

Patterson, M. L., Foster, J. L., \& Bellmer, C. D. (2001). Another look at accuracy and confidence in social judgments. Journal of Nonverbal Behavior, 25, 207-219.

Pozzulo, J. D., \& Warren, K. L. (2003). Descriptions and identifications of strangers by youth and adult eyewitnesses. Journal of Applied Psychology, 88, 315-323.

Roberts, T., \& Bruce, V. (1988). Feature saliency in judging the sex and familiarity of faces. Perception, 17, 475-481.

Rosenthal, R., \& Rosnow, R. L. (2007). Essentials of behavioral research: Methods and data analysis (3rd ed). New York: McGraw-Hill. 
Rudd, N. A. (1996). Appearance and self-presentation research in gay consumer cultures: Issues and impact. Journal of Homosexuality, 31, $109-134$

Schyns, P. G., Bonnar, L., \& Gosselin, F. (2002). Show me the features! Understanding recognition from the use of visual information. Psychological Science, 13, 402-409.

Shelp, S. (2002). Gaydar: Visual detection of sexual orientation among gay and straight men. Journal of Homosexuality, 44, 1-14.

Shepherd, J., Davies, G., \& Ellis, H. (1981). Studies of cue saliency. In G. Davies, H. Ellis, \& J. Shepherd (Eds.), Perceiving and remembering faces (pp. 105-131). London: Academic Press.

Smith, H. J., Archer, D., \& Costanzo, M. (1991). "Just a hunch": Accuracy and awareness in person perception. Journal of Nonverbal Behavior, 15, 3-18.

Smith, M. L., Cottrell, G. W., Gosselin, F., \& Schyns, P. G. (2005).
Transmitting and decoding facial expressions. Psychological Science, 16, 184-189.

Smyth, R., Jacobs, G., \& Rogers, H. (2003). Male voices and perceived sexual orientation: An experimental and theoretical approach. Language in Society, 32, 329-350.

Vinette, C., Gosselin, F., \& Schyns, P. G. (2004). Spatio-temporal dynamics of face recognition in a flash: It's in the eyes. Cognitive Science, 28, 289-301.

Wright, D. B., \& Sladden, B. (2003). An own gender bias and the importance of hair in face recognition. Acta Psychologica, 114, 101114

Received April 3, 2007

Revision received May 20, 2008

Accepted May 29, 2008

\section{New Editors Appointed, 2010-2015}

The Publications and Communications Board of the American Psychological Association announces the appointment of 4 new editors for 6-year terms beginning in 2010. As of January 1 , 2009, manuscripts should be directed as follows:

- Psychological Assessment (http://www.apa.org/journals/pas), Cecil R. Reynolds, PhD, Department of Educational Psychology, Texas A\&M University, 704 Harrington Education Center, College Station, TX 77843.

- Journal of Family Psychology (http://www.apa.org/journals/fam), Nadine Kaslow, PhD, Department of Psychiatry and Behavioral Sciences, Grady Health System, 80 Jesse Hill Jr. Drive, SE, Atlanta, GA 30303.

- Journal of Experimental Psychology: Animal Behavior Processes (http://www.apa.org/ journals/xan), Anthony Dickinson, PhD, Department of Experimental Psychology, University of Cambridge, Downing Street, Cambridge CB2 3EB, United Kingdom

- Journal of Personality and Social Psychology: Personality Processes and Individual Differences (http://www.apa.org/journals/psp), Laura A. King, PhD, Department of Psychological Sciences, University of Missouri, McAlester Hall, Columbia, MO 65211.

Electronic manuscript submission: As of January 1, 2009, manuscripts should be submitted electronically via the journal's Manuscript Submission Portal (see the website listed above with each journal title).

Manuscript submission patterns make the precise date of completion of the 2009 volumes uncertain. Current editors, Milton E. Strauss, PhD, Anne E. Kazak, PhD, Nicholas Mackintosh, $\mathrm{PhD}$, and Charles $\mathrm{S}$. Carver, $\mathrm{PhD}$, will receive and consider manuscripts through December 31, 2008. Should 2009 volumes be completed before that date, manuscripts will be redirected to the new editors for consideration in 2010 volumes. 